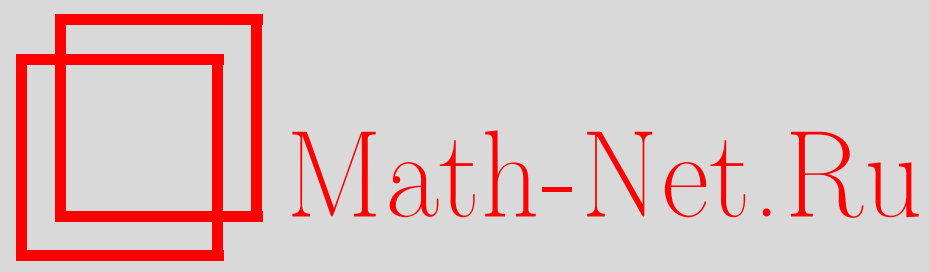

О. В. Пугачев, Построение негауссовских поверхностных мер методом Маллявэна, Матем. заметки, 1999, том 65, выпуск 3, 377-388

DOI: https://doi.org/10.4213/mzm1061

Использование Общероссийского математического портала Math-Net.Ru подразумевает, что вы прочитали и согласны с пользовательским соглашением http://www . mathnet.ru/rus/agreement

Параметры загрузки:

IP : 34.239 .49 .27

26 апреля 2023 г., 13:51:08 


\title{
ПОСТРОЕНИЕ НЕГАУССОВСКИХ ПОВЕРХНОСТНЫХ МЕР МЕТОДОМ МАЛЛЯВЭНА
}

\author{
O.В. Пугачёв
}

В работе обобщена теорема Эро-Маллявэна о существовании поверхностных мер на бесконечномерных пространствах с гауссовскими мерами на поверхностях. Доказана плотность множества емкостей, порожденных классами Соболева на бесконечномерных пространствах.

Библиографоия: 11 названий.

Известны два подхода к построению поверхностных мер в бесконечномерных пространствах. Первый подход, восходящий к работе А. В. Скорохода [1] и развитьй в работах А. В. Угланова [2], [3] (впоследствии усовершенствованный В. И. Богачёвьпм в работе [4]), основан на построении локальной поверхностной меры на достаточно малых окрестностях точек поверхности. Этот метод работает для весьма широкого класса пространств при минимальных требованиях на гладкость меры и поверхности. В работе [5] автору удалось обобщить конструкцию Угланова для случая, когда определяющая функция $F$ поверхности имеет непрерывные частные производные вдоль плотно вложенного гильбертова пространства, но непрерьвность самой $F$ при этом уже не требуется. Недостаток этого метода заключается в том, что из существования локальных поверхностных мер не всегда вытекает существование глобальной поверхностной меры.

Совершенно иной подход был предложен П. Маллявэном и реализован в работе [6] для случая винеровской меры (этот метод описан в [7, гл. 6]). В этом методе мера строится сразу на всей поверхности, а условия гладкости поверхности связаны не с геометрией объемлющего пространства, а с геометрией пространства Камерона-Мартина, и вместо дифференцируемости определяющей функции по Гато требуется ее принадлежность к соболевскому классу. Однако эти условия гладкости достаточно жесткие, к тому же в [6] и последующих работах на эту тему используются специфические свойства гауссовских мер. Цель данной работы - обобшить метод Маллявэна и построить поверхностную меру в случае более широкого класса пространств и поверхностей для достаточно гладких негауссовских мер.

1. Дифференцируемые меры и классы Соболева. Пусть $X$ - сепарабельное пространство Фреше (например, сепарабельное банахово пространство), в него непрерьвно вложено сепарабельное гильбертово пространство $H$. Обозначим через $\langle\cdot, \cdot\rangle$

Работа выполнена при поддержке Российского фонда фундаментальных исследований, гранты № 96-15-96865 и № 97-01-00932, и германского гранта № 436 RUS 113/343/0(R). 
скалярное произведение в $H$, а через $|\cdot|$ норму в $H$. Автоматически возникает непрерьвное отображение $j_{H}: X^{*} \rightarrow H^{*}=H$, заданное соотношением

$$
\left\langle j_{H}(l), h\right\rangle=l(h), \quad h \in H .
$$

Если $H$ плотно в $X$, то $j_{H}$ является вложением. Если $f$ дифференцируема по Гато, то ковектору $f^{\prime}(x) \in X^{*}$ соответствует вектор $\nabla f(x)=j_{H}\left(f^{\prime}(x)\right)$.

Будем обозначать через $F C_{b}^{\infty}(X)$ класс гладких ограниченных цилиндрических функций вида $\varphi(x)=u\left(l_{1}(x), \ldots, l_{k}(x)\right), l_{j} \in X^{*}, k \in \mathbb{N}, u \in C_{b}^{\infty}\left(\mathbb{R}^{k}\right) ; F C_{b}^{\infty}(X, H)$ - класс векторных полей $v(x)=\sum_{j=1}^{n} \varphi_{j}(x) e_{j}, \varphi_{j} \in F C_{b}^{\infty}(X),\left\{e_{j}\right\}$ - ортонормированный базис $H, n \in \mathbb{N}$.

Пусть $\mu$-вероятностная мера на $X$. Из полноты и сепарабельности метризуемого пространства $X$ вытекает, что $\mu$-радоновская мера (см. [8]).

ОПРЕДЕЛЕниЕ 1. Мера $\mu$ на $X$ назьвается дифферениируемой вдоль вектора $h \in X$ в смысле Фомина (далее это будет назьваться просто дифферениируемостью), если существует знакопеременная мера ограниченной вариации $d_{h} \mu$ такая, что для всех функций $\varphi \in F C_{b}^{\infty}(X)$ справедлива формула

$$
\int_{X} \partial_{h} \varphi(x) \mu(d x)=-\int_{X} \varphi(x) d_{h} \mu(d x)
$$

(формула интегрирования по частям) и при этом $d_{h} \mu$ абсолютно непрерывна относительно $\mu$.

Приведем также равносильное определение: $\mu$ дифференцируема вдоль $h$, если сушествует мера $d_{h} \mu$ такая, что $\left.\partial_{t}\right|_{t=0} \mu(B+t h)=d_{h} \mu(B)$ для всех $B \in \mathscr{B}(X)$.

Плотность меры $d_{h} \mu$ относительно $\mu$ будем обозначать через $\beta_{h}^{\mu}$ и называть логарифмической производной меры $\mu$ вдоль $h$.

Всюду далее мы будем предполагать меру $\mu$ таковой, что если функция $\varphi \in F C_{b}^{\infty}(X)$ равна $0 \mu$-почти всюду, то $\nabla \varphi=0 \mu$-почти всюду. В частности, это верно для меры с полным носителем. Будем также предполагать, что $\beta_{h}^{\mu} \in L^{2}(X, \mu)$ для всех $h \in H$.

Введем на пространствах $F C_{b}^{\infty}(X)$ и $F C_{b}^{\infty}(X, H)$ нормы:

$$
\|\varphi\|_{m, p}=\left(\int_{X}\left(N_{m, \varphi}(x)\right)^{p} \mu(d x)\right)^{1 / p}, \quad\|v\|_{m, p}=\left(\int_{X}\left(N_{m, v}(x)\right)^{p} \mu(d x)\right)^{1 / p},
$$

где

$$
N_{m, \varphi}^{2}(x)=|\varphi(x)|^{2}+\sum_{k=1}^{m} \sum_{i_{1}=1}^{\infty} \cdots \sum_{i_{k}=1}^{\infty}\left|\partial_{e_{i_{1}}} \cdots \partial_{e_{i_{k}}} \varphi(x)\right|^{2}
$$

и аналогичная формула справедлива для $N_{m, v}(x)$.

ОПРЕДЕЛЕНИЕ 2. Пополнив пространства $F C_{b}^{\infty}(X)$ и $F C_{b}^{\infty}(X, H)$ по указанным нормам $\|\cdot\|_{m, p}$, получим соболевские пространства $W^{m, p}(X)$ и $W^{m, p}(X, H)$ соответственно, где $m$ - целое неотрицательное, $1 \leqslant p<\infty$. При $p=\infty$ соответствующие пространства определяются как $W^{m, \infty}=\bigcap_{p \in \mathbb{N}} W^{m, p}$.

Нормы в пространствах $W^{0, p}=L^{p}$ будем обозначать без индекса 0 .

Заметим, что построенные пространства и нормы в них не зависят от выбора базиса в $H$. 
ОПредЕЛЕНИЕ 3 . Пусть $f \in W^{m, p}(X), h \in H$. Пусть $f_{n} \in F C_{b}^{\infty}(X), f_{n}$ сходятся к $f$ в $L^{p}(X, \mu)$ и $\left\|f_{n}-f_{k}\right\|_{m, p} \rightarrow 0$ при $n, k \rightarrow \infty$. Тогда предел в $L^{p}(X, \mu)$ функций $\partial_{h} f_{n}$ обозначим через $\partial_{h} f$ и будем назьвать производной (в смысле Соболева) функции $f$ вдоль вектора $h$.

Аналогично определяются кратные производные $f$ до порядка $m$.

Если $p \geqslant 2$, то $\partial_{h} f$ существует и не зависит от выбора последовательности $f_{n}$. Это доказьвается через формулу интегрирования по частям так же, как было доказано в [7, гл. 5] для случая гауссовских мер.

ЛЕмма 1. Если $f \in W^{m, p}(X), g \in W^{m, q}(X), 1 \leqslant p, q<\infty, 1 / p+1 / q=1 / r \leqslant 1$, mо $f g \in W^{m, r}(X)$, причем $\|f g\|_{m, r} \leqslant C(m, p, q, r) \cdot\|f\|_{m, p} \cdot\|g\|_{m, q}$.

Эта оценка выводится из неравенства Гёльдера сначала для гладких цилиндрических функций, а затем путем перехода к пределу для всех $f$ и $g$.

ОПРЕДЕЛЕНИЕ 4. Мера $\mu$ дифферениируема вдоль векторного поля $v$ (в смысле Фомина), если существует знакопеременная мера ограниченной вариации $d_{v} \mu$ такая, что для всякой $\varphi \in F C_{b}^{\infty}(X)$ справедливо равенство

$$
\int \partial_{v} \varphi(x) \mu(d x)=-\int \varphi(x) d_{v} \mu(d x),
$$

где $\partial_{v} \varphi(x)=\varphi^{\prime}(x)(v(x))$, и при этом $d_{v} \mu \ll \mu$.

Плотность меры $d_{v} \mu$ относительно $\mu$ будем назьвать дивергениией векторного поля $v$ и обозначать через $\delta v$.

Из леммы 1 можно получить оценки норм производных $\varphi$ вдоль $v$ через нормы $\varphi$ и $v$ (при условии, что $\varphi$ и $v$ принадлежат соответствующим соболевским классам):

$$
\begin{array}{ll}
\left\|\partial_{v} \varphi\right\|_{r} \leqslant C_{1}(p, q, r) \cdot\|\varphi\|_{1, p} \cdot\|v\|_{q}, & \text { где } \frac{1}{r} \geqslant \frac{1}{p}+\frac{1}{q}, \\
\left\|\partial_{v}^{k} \varphi\right\|_{r} \leqslant C_{k}(p, q, r) \cdot\|\varphi\|_{k, p} \cdot\|v\|_{k-1, q}^{k}, & \text { где } \frac{1}{r} \geqslant \frac{1}{p}+\frac{k}{q} .
\end{array}
$$

Лемма 2. Если $f \in W^{1,2}(X)$, а мера $\mu$ дифферениируема вдоль векторного поля $v \in L^{2}(X, \mu, H)$, причем $\delta v \in L^{2}(X, \mu)$, то мера $f \mu$ такжсе дифферениируема

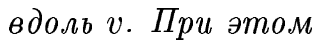

$$
d_{v}(f \mu)=\left(f \cdot \delta v+\partial_{v} f\right) \mu .
$$

ДокАЗАТЕЛЬСТво. Пусть сначала $f \in F C_{b}^{\infty}(X)$. Тогда для всякой $\varphi \in F C_{b}^{\infty}(X)$ имеем

$$
\begin{aligned}
\int \partial_{v} \varphi(x) f(x) \mu(d x) & =\int \partial_{v}(\varphi(x) f(x)) \mu(d x)-\int \varphi(x) \partial_{v} f(x) \mu(d x) \\
& =-\int \varphi(x) f(x) d_{v} \mu(d x)-\int \varphi(x) \partial_{v} f(x) \mu(d x) \\
& =-\int \varphi(x)\left(f(x) \delta v(x)+\partial_{v} f(x)\right) \mu(d x) .
\end{aligned}
$$

Вариация меры $\left(f \cdot \delta v+\partial_{v} f\right) \mu$ не превьшает $\|f\|_{2} \cdot\|\delta v\|_{2}+\|\nabla f\|_{2} \cdot\|v\|_{2}$, что при фиксированном $v$ оценивается через $\|f\|_{1,2}$. Благодаря этому формула (1) остается справедливой при переходе к пределу по $f$ в $W^{1,2}(X)$.

Введем для меры $\mu$ аналог пространства Камерона-Мартина. 
ОПРЕдЕлЕниЕ 5 . Обозначим через $H(\mu)$ пространство

$$
\left\{h \in X: \mu \text { дифференцируема вдоль } h \text { и }\left\|\beta_{h}^{\mu}\right\|_{2}<\infty\right\} \text {, }
$$

наделенное нормой $\|h\|_{H(\mu)}=\left\|\beta_{h}^{\mu}\right\|_{2}$.

$H(\mu)$ является сепарабельньг гильбертовым пространством, непрерьвно вложенным в $X$ (см. [9, гл. 5]).

\section{2. Абсолютная непрерывность образа меры.}

ТеОРема 1. Пусть выполнены следующие условия:

a) $F \in W^{2,2}(X)$;

б) $1 /|\nabla F| \in L^{4}(X, \mu)$;

в) мера $\mu$ дифферениируема вдоль $v=\nabla F u \delta v \in L^{2}(X, \mu)$.

Тогда образ меры $\mu \circ F^{-1}$ абсолютно непрерывен относительно меры Лебега на $\mathbb{R}$, причем плотность $k$ мохно выбрать с ограниченной вариацией.

ДокАЗАТЕльство. Для доказательства существования требуемой плотности достаточно существования меры $\lambda_{1}$ на $X$ такой, что для любой функции $\varphi \in C_{b}^{1}(\mathbb{R})$

$$
\int \varphi^{\prime}(t) \mu \circ F^{-1}(d t) \equiv \int \varphi^{\prime}(F(x)) \mu(d x)=-\int \varphi(F(x)) \lambda_{1}(d x) .
$$

Функция $\varphi$ о $F$ принадлежит классу $W^{1,2}(X)$ (см. [9, лемма 7.1.13]). Сделаем следуюшие преобразования:

$$
\begin{aligned}
\int \varphi^{\prime}(F(x)) \mu(d x) & =\int \varphi^{\prime}(F(x)) \partial_{v} F(x) \frac{\mu(d x)}{\partial_{v} F(x)}=\int \partial_{v}(\varphi(F(x))) \frac{\mu(d x)}{\partial_{v} F(x)} \\
& =-\int \varphi(F(x)) d_{v}\left(\frac{\mu}{\partial_{v} F}\right)(d x)
\end{aligned}
$$

(последнее равенство пока формально). Положим

$$
\lambda_{1}=d_{v}\left(\frac{\mu}{\partial_{v} F}\right)=\left(-\frac{\partial_{v}^{2} F}{|\nabla F|^{4}}+\frac{\delta v}{|\nabla F|^{2}}\right) \mu .
$$

Ограниченность вариации меры $\lambda_{1}$ доказьвается при помоши оценок через нормы: заметим, что

$$
\left|\partial_{v}^{2} F\right|=\left|2\left\langle\nabla^{2} F(\nabla F), \nabla F\right\rangle\right| \leqslant 2 N_{2, F} \cdot|\nabla F|^{2},
$$

откуда получается по неравенству Гёльдера

$$
\left\|\lambda_{1}\right\| \leqslant\left\|\frac{2 N_{2, F}+|\delta v|}{|\nabla F|^{2}}\right\|_{1} \leqslant\left(2\|F\|_{2,2}+\|\delta v\|_{2}\right)\left\|\frac{1}{|\nabla F|}\right\|_{4}^{2}<\infty .
$$

Поскольку функция $\partial_{v}(\varphi \circ F)$ интегрируема по $\left(\partial_{v} F\right)^{-1} \mu$, а ограниченная функция $\varphi \circ F$ интегрируема по $\lambda_{1}$, то проделанные преобразования законны. В результате мы имеем дифференцируемость меры $\mu \circ F^{-1}$ на $\mathbb{R}$ в смысле Скорохода, $d_{1}\left(\mu \circ F^{-1}\right)=\lambda_{1} \circ F^{-1}$. Из этого следует (см. [9, пример 2.2.1]), что некоторая версия лебеговой плотности меры $\mu \circ F^{-1}$ имеет конечную вариацию (не превышающую $\left\|\lambda_{1}\right\|$ ), а значит, имеет не более чем счетное множество точек разрыва, причем только первого рода.

Всюду далее мы будем рассматривать версию функции $k$, имеющую конечную вариацию и определенную в каждой точке разрьва $a$ таким образом, что

$$
k(a)=\frac{k(a-0)+k(a+0)}{2} .
$$


ЗАмЕчАниЕ 1. Справедливо неравенство

$$
\sup _{t}|k(t)| \leqslant \frac{1}{2} \operatorname{Var} k \leqslant \frac{1}{2}\left\|\lambda_{1}\right\|
$$

Это следует из очевидного факта, что inf $k=0$.

СледСтвиЕ 1. Пусть в теореме $1|\nabla F|^{-1} \in L^{p}(X, \mu)$, где $p>4$. Пусть $g \in W^{1, r}(X)$, причем константы $p$ и $r$ удовлетворяют неравенству $2 / p+1 / r \leqslant 1 / 2$. Тогда мера $g \mu$ дифферениируема вдоль $v=\nabla F$, и ее образ $(g \mu) \circ F^{-1}$ имеет плотность $k_{g}$ с ограниченной вариачией.

Это утверждение доказывается построением меры $\lambda_{g}$ по мере $g \mu$ с помощью той же формулы, что и в теореме 1 . Оценим $\sup _{t}\left|k_{g}(t)\right| \leqslant\left\|\lambda_{g}\right\|$ :

$$
\begin{gathered}
\lambda_{g}=d_{v}\left(\frac{g \mu}{\partial_{v} F}\right)=\left(-\frac{\partial_{v}^{2} F}{|\nabla F|^{4}} g+\frac{\partial_{v} g}{|\nabla F|^{2}}+\frac{g \delta v}{|\nabla F|^{2}}\right) \mu, \\
\left\|\lambda_{g}\right\| \leqslant\left\|\frac{\left(2 N_{2, F}+|\delta v|\right)|g|+|\langle\nabla F, \nabla g\rangle|}{|\nabla F|^{2}}\right\|_{1}
\end{gathered}
$$

откуда по неравенству Гёльдера получаем

$$
\left\|\lambda_{g}\right\| \leqslant C(\mu, F, p, r) \cdot\|g\|_{1, r} .
$$

Для функции $k_{g}$ мы также будем выбирать версию конечной вариации со свойством (2).

СлеДСтвиЕ 2. Пусть выполнены условия следствия 1. Пусть $\omega_{1}-$ положительная четная $C^{\infty}$-функиия с носителем $[-1,1], \int \omega_{1}(t) d t=1, \omega_{N}(t)=N \omega_{1}(N t)$. Тогда для всех $g$, удовлетворятои их условиям следствия 1 , имеем

$$
\int_{X} g(x) \omega_{N}(F(x)) \mu(d x)=\int_{-\infty}^{+\infty} k_{g}(t) \omega_{N}(t) d t \rightarrow k_{g}(0) \quad \text { npu } \quad N \rightarrow \infty .
$$

ЛЕмма 3. Если $X$ - полное локально выпуклое пространство, $\mu$-радоновская вероятностная мера на нем, то существуют выпуклые чентрально-симметричные компакты $K_{t}$, исчерпьвающие меру, т.е. для всех $t \geqslant 1 \mu\left(K_{t}\right) \geqslant 1-1 / t u$ $K_{s} \subset K_{t}$ npu $s<t$.

Это вытекает из того, что замкнутая абсолютно вьпуклая оболочка компакта в полном линейном топологическом пространстве также является компактом.

ОПРЕДЕЛЕнИЕ 6 . Пусть $m \geqslant 0,1 \leqslant p<\infty$. Емкость Сар $m, p$ открытого множества $U$ равна

$$
\operatorname{Cap}_{m, p}(U)=\inf \left\{\|\varphi\|_{m, p}: \varphi \in W^{m, p}(X), \varphi \geqslant 0, \varphi \geqslant 1 \text { на } \mu \text {-почти всем } U\right\},
$$

для произвольного множества $A$

$$
\operatorname{Cap}_{m, p}(A)=\inf \left\{\operatorname{Cap}_{m, p}(U): U \text { открыто, } A \subset U\right\} .
$$




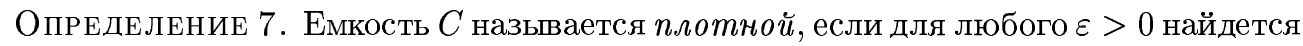
компакт $K_{\varepsilon} \subset X$ такой, что $C\left(X \backslash K_{\varepsilon}\right)<\varepsilon$.

Известно, что если $X$ - сепарабельное банахово пространство, $\mu$ - вероятностная

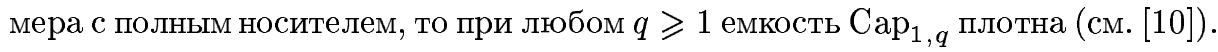

То же будет справедливо и для произвольного локально выпуклого пространства $X$, если $\mu$-радоновская гауссовская мера (см. [7, гл. 5]).

ЛЕмма 4. Пусть $\mu$ - вероятностная мера на сепарабельном пространстве Фреше $X, H \subset H(\mu)$. Тогда существует семейство функиий $\left\{\theta_{t} \mid t \geqslant 4\right\}$ из класса $W^{1, \infty}(X)$ со следуюш,ими свойствами:

1) $0 \leqslant \theta_{t} \leqslant 1$

2) $\theta_{t}(x) \rightarrow 1$ nри $t \rightarrow \infty$ для $\mu$-почти всех $x$;

3) $\theta_{t}=0$ н-почти всюду вне $2 K_{t}$, где $K_{t}$ - выпуклые симметричные компактыл, исчерпывающие меру;

4) для любого $q \geqslant 1\left\|1-\theta_{t}\right\|_{1, q} \rightarrow 0$ при $t \rightarrow \infty$.

В частности, из этого будет вытекать плотность емкости Сар $\mathrm{Ca}_{1, q}$ при всех $q$.

ДокАЗАТЕЛЬСтво. Пусть $C_{H}$ - норма оператора вложения $H$ в $H(\mu)$. Обозначим через $\mu_{\text {sym }}$ симметризованную меру $\mu$, т.е. вероятностную меру, заданную формулой $\mu_{\mathrm{sym}}(A)=(\mu(A)+\mu(-A)) / 2$. Введем функцию $\psi_{t}(x)=\mu_{\mathrm{sym}}\left(2 K_{t}-x\right)$. Тогда при $h \in H(\mu)$

$$
\begin{aligned}
\partial_{h} \psi_{t}(x) & =\frac{1}{2}\left(d_{h} \mu\left(2 K_{t}+x\right)-d_{h} \mu\left(2 K_{t}-x\right)\right)=\frac{1}{2}\left(\int_{2 K_{t}+x}-\int_{2 K_{t}-x}\right) \beta_{h}^{\mu}(y) \mu(d y) \\
& =\left(\frac{1}{2}\left(I_{X \backslash\left(2 K_{t}-x\right)}-I_{X \backslash\left(2 K_{t}+x\right)}\right) ; \beta_{h}^{\mu}\right)_{L^{2}(\mu)}
\end{aligned}
$$

Подставив $h=e_{n}, n=1,2, \ldots$, где $\left\{e_{n}\right\}$ - ортонормированньй базис $H(\mu)$, и просуммировав квадраты выражений (3), из неравенства Бесселя получим оценку

$$
\begin{gathered}
\left\|\nabla_{H(\mu)} \psi_{t}(x)\right\|_{H(\mu)} \leqslant\left\|I_{X \backslash\left(2 K_{t}+x\right)}\right\|_{2}+\left\|I_{X \backslash\left(2 K_{t}-x\right)}\right\|_{2}, \\
\left|\nabla \psi_{t}(x)\right| \leqslant C_{H} \cdot\left\|\nabla_{H(\mu)} \psi_{t}(x)\right\|_{H(\mu)} .
\end{gathered}
$$

При $x \notin 2 K_{t}$ имеем $\left(2 K_{t}-x\right) \cap\left(2 K_{t}+x\right)=\varnothing$, откуда $\mu_{\text {sym }}\left(2 K_{t}-x\right)=\mu_{\text {sym }}\left(2 K_{t}+x\right) \leqslant 1 / 2$. При $x \in K_{t} \mu_{\text {sym }}\left(2 K_{t}-x\right) \geqslant \mu_{\text {sym }}\left(K_{t}\right) \geqslant 1-t^{-1} \geqslant 3 / 4$. Кроме того, для любого $x$ $0 \leqslant \psi_{t}(x) \leqslant 1$. Оценим норму функции $\psi_{t}$. При $x \in K_{t}$ имеем $X \backslash\left(2 K_{t} \pm x\right) \subset X \backslash K_{t}$; следовательно,

$$
\int\left|\nabla \psi_{t}(x)\right|^{q} \mu(d x) \leqslant \int_{K_{t}} C_{H}^{q}\left(\frac{2}{\sqrt{t}}\right)^{q} \mu(d x)+\int_{X \backslash K_{t}} C_{H}^{q} \mu(d x)
$$

правая часть этого неравенства стремится к 0 при $t \rightarrow \infty$. Наконец, $\psi_{t}$ приближается по норме $\|\cdot\|_{1, q}$ функциями $f_{n}(x)=\mu_{\text {sym }}\left(E_{n}-x\right)$, где $E_{n}$ - абсолютно вьпуклые замкнутые цилиндры, убьваюшие к компакту $2 K_{t}$ (их существование вытекает из того, что $X$ - сепарабельное пространство $\Phi$ реше). $Ф$ ункции $f_{n}$ являются цилиндрическими и липшицевыми вдоль $H$; следовательно, $f_{n} \in W^{1, \infty}(X)$. 
Таким образом, $\psi_{t} \in W^{1, q}(X)$. Пусть монотонная функция $f \in C_{b}^{\infty}(\mathbb{R})$ такова, что $f(t)=0$ при $t \leqslant 1 / 2, f(t)=1$ при $t \geqslant 3 / 4$. Пусть $\theta_{t}(x)=f\left(\psi_{t}(x)\right)$. Тогда $\theta_{t}$ обладает всеми необходимьми свойствами: $0 \leqslant \theta_{t} \leqslant 1, \theta_{t}=1$ на $K_{t}, \theta_{t}=0$ вне $2 K_{t}$;

$$
\left\|1-\theta_{t}\right\|_{q} \leqslant t^{-1 / q}, \quad\left\|\nabla \theta_{t}\right\|_{q} \leqslant \sup _{\tau} f^{\prime}(\tau) \cdot\left\|\nabla \psi_{t}\right\|_{q} \rightarrow 0 \text { при } t \rightarrow \infty .
$$

ОПРЕДЕЛЕНИЕ 8. Пусть $C$ - некоторая емкость. Будем говорить, что:

1) некоторое свойство выполнено $C$-квазивсюду, если оно имеет место на $X \backslash A$, где $C(A)=0$

2) функция $f C$-квазинепрерывна, если существуют замкнутые множества $Q_{n}$ такие, что $\left.f\right|_{Q_{n}}$ непрерывна для всех $n$ и $C\left(X \backslash Q_{n}\right)<1 / n$.

Заметим, что если емкость $C$ плотна, то множества $Q_{n}$ можно выбрать компактными.

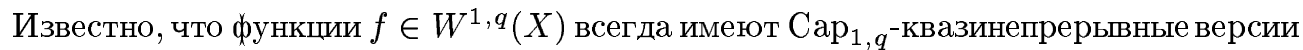
(см. [7, гл. 5]).

\section{3. Построение и свойства поверхностной меры.}

Лемма 5. Пусть $\mu$-вероятностная мера на сепарабельном пространстве Фреше $X ; F \in W^{2,2}(X),|\nabla F|^{-1} \in L^{8}(X, \mu)$. Пусть $H \subset H(\mu), \mu$ дифференцируема вдоль $v=\nabla F$, причем $\delta v \in L^{2}(X, \mu)$. Тогда

1) мерьи

$$
\nu_{N}^{t}(d x)=\omega_{N}(F(x)) \theta_{t}(x) \mu(d x)
$$

$\left(\omega_{N}\right.$ и $\theta_{t}$ были определены в $\left.n .2\right)$ слабо сходятся при $N \rightarrow \infty$ к некоторой мере $\nu^{t}$, сосредоточенной на $2 K_{t}$;

2) если $\operatorname{Cap}_{1,4}(A)=0$, то для всех $t$ и $N \quad \nu_{N}^{t}(A)=0, \nu^{t}(A)=0$;

3) для всех $g \in F C_{b}^{\infty}(X)$ выполнено равенство

$$
\int g(x) \nu^{t}(d x)=k_{g \theta_{t}}(0)
$$

4) равенство (4) справедливо и для всех $\operatorname{Cap}_{1,8}$-квазинепрерывных $g \in W^{1,8}(X)$.

ДокаЗАтельство. 1) Пусть $\varphi \in C_{b}(X)$. Зададим $\varepsilon>0$. На компакте $2 K_{t}$ функция $\varphi$ равномерно непрерывна. Поэтому в силу теоремы Вейерштрасса существует функция $\varphi_{\varepsilon} \in F C_{b}^{\infty}(X)$ такая, что

$$
\sup _{x \in 2 K_{t}}\left|\varphi_{\varepsilon}(x)-\varphi(x)\right| \leqslant \varepsilon
$$

Следовательно,

$$
\lim _{\varepsilon \rightarrow 0} \int \varphi_{\varepsilon}(x) \nu_{N}^{t}(d x)=\int \varphi(x) \nu_{N}^{t}(d x)
$$

равномерно при всех $N$, поскольку меры $\nu_{N}^{t}$ равномерно ограничены:

$$
\nu_{N}^{t}(X)=\int_{-\infty}^{+\infty} k_{\theta_{t}}(\tau) \omega_{N}(\tau) d \tau \rightarrow k_{\theta_{t}}(0)
$$


При каждом $\varepsilon$ имеем $\varphi_{\varepsilon} \theta_{t} \in W^{1,8}(X)$ и по следствию 2 существует

$$
\lim _{N \rightarrow \infty} \int \varphi_{\varepsilon}(x) \nu_{N}^{t}(d x)=k_{\varphi_{\varepsilon} \theta_{t}}(0)
$$

Отсюда следует, что существует

$$
\lim _{N \rightarrow \infty} \int \varphi(x) \nu_{N}^{t}(d x)
$$

Поскольку это верно для всех $\varphi \in C_{b}(X)$ и при этом меры $\nu_{N}^{t}$ равномерно плотны, по теореме Прохорова (см. $\left[11\right.$, гл. I]) сушествует мера $\nu^{t}$, к которой слабо сходятся меры $\nu_{N}^{t}$. По свойству слабо сходящихся мер для открытого множества (см. [8, гл. 1])

$$
\nu^{t}\left(X \backslash 2 K_{t}\right) \leqslant \lim _{N \rightarrow \infty} \nu_{N}^{t}\left(X \backslash 2 K_{t}\right)=0
$$

2) Пусть $\operatorname{Cap}_{1,4}(A)=0$. Для каждого натурального $m$ возьмем открытое, содержащее $A$, множество $U_{m}$ такое, что $\operatorname{Cap}_{1,4}\left(U_{m}\right)<2^{-m}$. Существует функция $g_{m} \in W^{1,4}(X)$ такая, что $g_{m} \geqslant 0, g_{m} \geqslant 1$ на $\mu$-почти всем (следовательно, и на $\nu_{N}^{t}$-почти всем) $U_{m}$, $\left\|g_{m}\right\|_{1,4}<2^{1-m}$. При всех $N$ имеем

$$
\begin{aligned}
\nu_{N}^{t}\left(U_{m}\right) \leqslant & \int g_{m}(x) \nu_{N}^{t}(d x)=\int_{-\infty}^{+\infty} k_{g_{m} \theta_{t}}(\tau) \omega_{N}(\tau) d \tau \leqslant \underset{\tau}{\operatorname{ess} \sup _{\tau}\left|k_{g_{m} \theta_{t}}(\tau)\right|,} \\
& \underset{\tau}{\operatorname{ess} \sup }\left|k_{g_{m} \theta_{t}}(\tau)\right| \leqslant \sup _{\tau}\left|k_{g_{m}}(\tau)\right| \leqslant C(\mu, F, 8,4) \cdot\left\|g_{m}\right\|_{1,4}
\end{aligned}
$$

по следствию 1 . Следовательно, для всех $t, N \nu_{N}^{t}\left(U_{m}\right) \leqslant$ const $/ 2^{m}$. По свойству слабо сходящихся мер имеем

$$
\nu^{t}\left(U_{m}\right) \leqslant \varliminf_{N \rightarrow \infty} \nu_{N}^{t}\left(U_{m}\right) \leqslant \frac{\text { const }}{2^{m}}, \quad \nu^{t}(A) \leqslant \inf _{m} \nu^{t}\left(U_{m}\right)=0 .
$$

$3), 4)$ Пусть $g \in W^{1,8}(X)$. Поскольку функции $g_{ \pm}(x)=\max \{ \pm g(x), 0\}$ также принадлежат $W^{1,8}(X)$, достаточно ограничиться рассмотрением неотрицательных $g$. По лемме 1 из $\theta_{t} \in W^{1,8}(X)$ получаем $g \theta_{t} \in W^{1,4}(X)$. В случае непрерывности (и ограниченности) $g$ на $2 K_{t}$ утверждение непосредственно вытекает из существования предела

$$
\lim _{N \rightarrow \infty} \int g \nu_{N}^{t}=k_{g \theta_{t}}(0) .
$$

Общий случай сводится к этому случаю в силу замечаний 2 и 3 (см. ниже), в виду того, что функция $g$ имеет Сар 1,8 -квазинепрерьвную версию и множества $Q_{n}$ из определения 8 можно выбрать компактными.

ЗАмечАниЕ 2. Поскольку емкость Сар 1,8 плотна, возрастающие компакты $K_{t}^{\prime}$ можно выбрать такими (не обязательно центрально симметричньпи и вьпуклыми), что $\operatorname{Cap}_{1,8}\left(X \backslash K_{t}^{\prime}\right)<1 / t$. В этом случае существуют функции $\theta_{t}: X \rightarrow[0,1]$ со свойствами:
а) $\theta_{t}=0 \mu$-почти всюду на $X \backslash K_{t}^{\prime}$;
б) $\theta_{t} \rightarrow 1$ при $t \rightarrow \infty \mu$-почти всюду;
в) $\theta_{t} \in W^{1,8}(X)$;
г) $\left\|1-\theta_{t}\right\|_{1,8} \rightarrow 0$ при $t \rightarrow \infty$. 
Лемма 6. Пусть $\nu^{t}-$ меры, построенные в предьдущей лемме. Тогда

1) меры $\nu^{t}$ возрастают по $t$;

2) меры $\nu^{t}$ слабо сходятся $к$ мере $\nu$ и сходятся $к$ ней же по вариации, $\|\nu\|=k(0)$; в частности, для всех $g \in F C_{b}^{\infty}(X)$ выполнено равенство

$$
\int g(x) \nu(d x)=k_{g}(0)
$$

3) если $\operatorname{Cap}_{1,4}(A)=0$, mo $\nu(A)=0$;

4) если $g \in W^{1,8}(X)$ является Сар ${ }_{1,8}$-квазинепрерывной, то выполнено равенство (5).

ДокАЗАТЕЛЬСтво. 1) Докажем, что при $t>s \geqslant 4$ для любой ограниченной неотрицательной борелевской функции $\varphi$ имеем

$$
\int \varphi \nu^{t} \geqslant \int \varphi \nu^{s}
$$

Достаточно проверить (6) для любой функции $\varphi \in F C_{b}^{\infty}(X), \varphi \geqslant 0$. А это вытекает из следствия 2 , поскольку $\varphi \theta_{t} \geqslant \varphi \theta_{s}$.

2) Пусть $\varphi \in C_{b}(X), \sup |\varphi(x)|=1, t>s \geqslant 4$. Имеем

$$
\begin{aligned}
\left|\int \varphi \nu^{t}-\int \varphi \nu^{s}\right| & =\lim _{N \rightarrow \infty}\left|\int \varphi \nu_{N}^{t}-\int \varphi \nu_{N}^{s}\right| \leqslant \lim _{N \rightarrow \infty} \int\left|\nu_{N}^{t}-\nu_{N}^{s}\right| \\
& =\lim _{N \rightarrow \infty} \int\left|k_{\theta_{t}}(\tau)-k_{\theta_{s}}(\tau)\right| \omega_{N}(\tau) d \tau=\left|k_{\theta_{t}}(0)-k_{\theta_{s}}(0)\right| .
\end{aligned}
$$

Поскольку $k_{\theta_{s}}(0) \nearrow k(0)$ при $s \rightarrow \infty$, мы получаем, что искомая разность равномерно по $t \geqslant s$ стремится к 0 при $s \rightarrow \infty$, т.е. выполнен критерий Коши для слабой сходимости мер $\nu^{t}$. Равномерная плотность мер $\nu^{t}$ вытекает из равномерных оценок $\nu^{t}$ через $\mathrm{Cap}_{1,4}$, полученных в лемме 5, поскольку Сар ${ }_{1,4}\left(X \backslash 2 K_{s}\right) \rightarrow 0$ при $s \rightarrow \infty$. Следовательно, в силу теоремы Прохорова эти меры слабо сходятся к некоторой мере $\nu$. При этом вариация меры $\nu$ равна

$$
\|\nu\|=\int \nu(d x)=\lim _{t \rightarrow \infty} \int \nu^{t}(d x)=\lim _{t \rightarrow \infty} k_{\theta_{t}}(0)=k(0) .
$$

Докажем сходимость по вариации:

$$
\begin{aligned}
\left\|\nu-\nu^{t}\right\| & =\sup \left\{\int \psi \cdot\left(\nu-\nu^{t}\right): \psi \text { борелевская, }|\psi| \leqslant 1\right\} \\
& =\sup \left\{\int \varphi \cdot\left(\nu-\nu^{t}\right): \varphi \in F C_{b}^{\infty},|\varphi| \leqslant 1\right\},
\end{aligned}
$$

так как в силу радоновости мер $\nu$ и $\nu^{t}$ функция $\psi$ приближается в $L^{1}\left(X,|\nu|+\left|\nu^{t}\right|\right)$ цилиндрическими функциями (см. [7, дополнения]). Если $\varphi \in F C_{b}^{\infty},|\varphi| \leqslant 1$, то

$$
\left|\int \varphi \nu-\int \varphi \nu^{t}\right|=\left|\lim _{s \rightarrow \infty} k_{\varphi \theta_{s}}(0)-k_{\varphi \theta_{t}}(0)\right|=\left|k_{\left(1-\theta_{t}\right) \varphi}(0)\right| \leqslant\left|k_{1-\theta_{t}}(0)\right| \rightarrow 0
$$


при $t \rightarrow \infty$.

3) Пусть для любого $m$ существует открытое множество $U_{m}$, содержащее $A$, такое, что $\operatorname{Cap}_{1,4}\left(U_{m}\right)<2^{-m}$. Как уже было показано, $\nu^{t}\left(U_{m}\right) \leqslant$ const $\cdot 2^{-m}$ при всех $t$. При каждом $m$ имеем

$$
\nu(A) \leqslant \nu\left(U_{m}\right) \leqslant \lim _{t \rightarrow \infty} \nu^{t}\left(U_{m}\right) \leqslant \text { const } \cdot 2^{-m} ;
$$

следовательно, $\nu(A)=0$.

4) Пусть $g \in W^{1,8}(X),\left.g\right|_{Q_{n}}$ непрерьвна для любого $n$, где замкнутые множества $Q_{n}$ возрастают и $\operatorname{Cap}_{1,8}\left(X \backslash Q_{n}\right) \rightarrow 0$. В силу плотности емкости Сар $\operatorname{Ca}_{1,8}$ мы можем считать $Q_{n}$ компактами. Поскольку $\mu\left(X \backslash Q_{n}\right) \rightarrow 0$, мы можем выбрать $Q_{n(t)}$ в качестве компактов $K_{t}^{\prime}$, где $n(t) \rightarrow \infty$ при $t \rightarrow \infty$ (см. замечание 2$)$. Тогда при каждом $t$ получаем

$$
\int g \nu^{t}=k_{g \theta_{t}}(0)
$$

Как уже было замечено, достаточно ограничиться рассмотрением неотрищательных $g$. На $Q_{n}$ функция $g$ интегрируема, так как она ограничена. Пусть $Q=\bigcup_{n=1}^{\infty} Q_{n}$. Тогда $\operatorname{Cap}_{1,4}(X \backslash Q) \leqslant \operatorname{Cap}_{1,8}(X \backslash Q)=0$; следовательно, $\nu(X \backslash Q)=0$. Отсюда мы получаем

$$
\lim _{n \rightarrow \infty} \int_{Q_{n}} g \nu=\int g \nu \leqslant \limsup _{s \rightarrow \infty} \int g \nu^{s} \leqslant \text { const } \cdot\|g\|_{1,4}<\infty .
$$

Следовательно, для любого $\varepsilon>0$ существует $n$ такое, что

$$
\left|\int g \nu-\int_{Q_{n}} g \nu\right|<\frac{\varepsilon}{3}
$$

и такая же оценка справедлива для мер $\nu^{s}$ равномерно при всех $s$; существует $s_{0} \geqslant 4$ такое, что

$$
\left|\int_{Q_{n}} g \nu-\int_{Q_{n}} g \nu^{s}\right| \leqslant\left\|\nu-\nu^{s}\right\| \cdot \sup _{Q_{n}} g<\frac{\varepsilon}{3} \quad \text { для всех } s \geqslant s_{0} .
$$

Тогда

$$
\left|\int g \nu-\int g \nu^{s}\right| \leqslant \varepsilon
$$

Следовательно,

$$
\int g \nu=\lim _{s \rightarrow \infty} \int g \nu^{s}=k_{g}(0)
$$

Теорема 2. Пусть $X$ - сепарабельное пространство Фреше (например, сепарабельное банахово пространство), $\mu$ - положительная мера, $H \subset H(\mu), F \in$ $W^{2,2}(X),|\nabla F|^{-1} \in L^{8}(X, \mu)$. Пусть мера $\mu$ дифферениируема вдоль векторного поля $v=\nabla F$, причем $\delta v \in L^{2}(X, \mu)$. Тогда существует единственная мера $\nu$ со свойством

$$
\int \varphi(x) \nu(d x)=k_{\varphi}(0) \quad \text { для всех } \varphi \in F C_{b}^{\infty}(X) .
$$


ДокАЗАтЕльство. Существование такой меры доказано ее построением. Единственность следует из того, что радоновская мера однозначно определяется интегралами цилиндрических функций. Заметим, что в доказательстве этой теоремы не использовались утверждения 4) лемм 5 и 6.

ЗАмЕчАниЕ 3. Результат построения меры $\nu$ не зависит от выбора компактов, исчерпывающих меру, и от выбора функций $\theta_{t}$ и $\omega_{N}$, обладающих вьшеописанными свойствами.

ЗАмЕчаниЕ 4. Если пространство $X$ непрерьвно вложено в сепарабельноепространство $\Phi$ реше $X^{\prime}$, функция $F$ произвольно продолжена вне $X$ и мера $\mu$ сосредоточена на $X$, то результат построения поверхностной меры не будет зависеть от того, какое из пространств, $X$ или $X^{\prime}$, рассматривать как объемлющее.

Введем новые обозначения. Пусть $\nu^{(0)} \equiv \nu, \nu^{(a)}$ - аналогичные меры, построенные для функций $F-a$. При всех $a$ таких, что $k(a) \neq 0$, положим $\mu_{\sigma}^{(a)}=\nu^{(a)} / k(a)$; если $k(a)=0$, положим $\mu_{\sigma}^{(a)} \equiv 0$.

ТЕОремА 3. Пусть выполнены те же условия на $X, H, \mu$ и $F$, что и в лемме 5 . Пусть $F \mathrm{Cap}_{1,4}$-квазинепрерывна. Тогда меры $\mu_{\sigma}^{(a)}$ являются условными мерами для $\mu$ относительно функиии $F$, т.e.

1) при $\mu \circ F^{-1}-$ почти всех а меры $\mu_{\sigma}^{(a)}$ вероятностные;

2) мера $\mu_{\sigma}^{(a)}$ сосредоточена на поверхности $F^{-1}(a)$;

3) для всех $B \in \mathscr{B}(X)$

$$
\mu(B)=\int_{-\infty}^{+\infty} \mu_{\sigma}^{(a)}(B) \mu \circ F^{-1}(d a)
$$

ДокАЗАтЕльСтво. 1) При $k(a) \neq 0\left\|\mu_{\sigma}^{(a)}\right\|=\left\|\nu^{(a)}\right\| / k(a)=1$.

2) Зафиксируем $a$ такое, что $k(a) \neq 0$. Пусть $Q_{n}$ - замкнутые множества такие, что $\left.F\right|_{Q_{n}}$ непрерывна и $\operatorname{Cap}_{1,4}\left(X \backslash Q_{n}\right)<1 / n$. Пусть $U=F^{-1}((-\infty, a-\delta) \cup(a+\delta,+\infty))$. Заметим, что

$$
U \subset \bigcap_{n=1}^{\infty} U_{n}
$$

где $U_{n}=U \cup\left(X \backslash Q_{n}\right)$ открытые, поскольку множества $F^{-1}([a-\delta, a+\delta]) \cap Q_{n}$ замкнуты. При всех $N>1 / \delta, t \geqslant 4$ имеем

$$
\left(\nu^{(a)}\right)_{N}^{t}\left(U_{n}\right)=\left(\nu^{(a)}\right)_{N}^{t}\left(X \backslash Q_{n}\right) \leqslant \text { const } \cdot \operatorname{Cap}_{1,4}\left(X \backslash Q_{n}\right)=\frac{\text { const }}{n}
$$

откуда

$$
\left(\nu^{(a)}\right)^{t}\left(U_{n}\right) \leqslant \frac{\mathrm{const}}{n}, \quad \mu_{\sigma}^{(a)}\left(U_{n}\right) \leqslant \frac{\mathrm{const}}{n k(a)}, \quad \mu_{\sigma}^{(a)}(U) \leqslant \inf _{n} \mu_{\sigma}^{(a)}\left(U_{n}\right)=0 .
$$


3) Пусть $\varphi(x)=e^{i l(x)}, l \in X^{*}$. Заметим, что при всех $a$, при которых $k$ и $k_{\varphi}$ непрерывны, $\left|k_{\varphi}(a)\right| \leqslant k(a)$. Обозначим через $J$ множество точек разрьва $k$ и $k_{\varphi}$, объединенное с множеством $\{k=0\}$. Тогда $\mu \circ F^{-1}(J)=0$ и

$$
\begin{aligned}
\int_{\mathbb{R}} k(a) \int_{X} \varphi(x) \mu_{\sigma}^{(a)}(d x) d a & =\int_{\mathbb{R} \backslash J} k(a) \frac{k_{\varphi}(a)}{k(a)} d a=\int_{\mathbb{R}} k_{\varphi}(a) d a \\
& =\int_{\mathbb{R}}(\varphi \mu) \circ F^{-1}(d a)=\int_{X} \varphi \mu(d x) .
\end{aligned}
$$

Преобразования Фурье радоновских мер $\int k(a) \mu_{\sigma}^{(a)} d a$ и $\mu$ совпадают, следовательно, совпадают сами эти меры.

ЗАмЕЧАнИЕ 5. Все приведенные доказательства остаются справедливьми для радоновской вероятностной меры на вполне регулярном локально вьпуклом пространстве, не являющемся сепарабельным пространством Фреше, если оно обладает следующим свойством: каждьй компакт содержится в некотором вьпуклом центрально симметричном компакте, представимом в виде счетного пересечения цилиндров (этим свойством обладает большинство пространств, встречающихся в приложениях).

При этом мера $\nu$ будет радоновской.

ЗАмЕчАнИЕ 6 . В доказательстве теоремы 1 и следствий 1,2 не использовался тот факт, что мера $\mu$ положительна. Леммы 5,6 (кроме утверждения 1 )) и теоремы 2,3 также легко обобщаются на случай знакопеременной меры. Разумеется, при построении соболевских классов, емкостей и функций $\theta_{t}$ меру $\mu$ следует заменить мерой $|\mu|$, которая обладает теми же свойствами дифференцируемости, что и $\mu$ (см. [9, гл. 2]). Кроме того, в случае знакопеременной $\mu k(t)$ будет обозначать лебегову плотность меры $|\mu| \circ F^{-1}$.

Автор выражает благодарность В. И. Богачёву за постановку задачи и научное руководство.

\section{СПИСОК ЦИТИРОВАННОЙ ЛИТЕРАТУРЫ}

[1] Скороход А. В. Интегрирование в гильбертовом пространстве. М.: Наука, 1975.

[2] Угланов А. В. Поверхностные меры в банаховом пространстве // Матем. сб. 1979. Т. 110. № 2. C. 189-217.

[3] Угланов А. В. Поверхностные интегралы в линейных топологических пространствах // Докл. РАН. 1995. Т. 344. № 4. С. 450-453.

[4] Bogachev V.I. Smooth measures, the Malliavin calculus and approximations in infinitedimensional space // Acta Univ. Carolin. Math. Phys. 1990. V. 31. № 2. P. 9-23.

[5] Пугачёв О.В. Поверхностные меры в бесконечномерных пространствах // Матем. заметки. 1998. Т. 63. №1. С. 106-114.

[6] Airault H., Malliavin P. Integration géométrique sur l'espace de Wiener // Bull. Sci. Math. (2). 1988. V. 112. P. 3-52.

[7] Богачёв В. И. Гауссовские меры. М.: Наука, 1997.

[8] Биллингсли П. Сходимость вероятностных мер. М.: Наука, 1977.

[9] Bogachev V. I. Differentiable measures and the Malliavin calculus // J. Math. Sci. 1997. V. 87. № 5. P. 3577-3731.

[10] Röckner M., Schmuland B. Tightness of general $C_{1, p}$ capacities on Banach space // J. Funct. Anal. 1992. V. 108. № 1. P. 1-12.

[11] Вахания Н. Н., Тариеладзе В. И., Чобанян С. А. Вероятностные распределения в банаховых пространствах. М.: Наука, 1984. 